Теорія Ймовір. та Матем. Статист. Вип. 78, 2008
Theor. Probability and Math. Statist.

No. 78, 2009, Pages 75-82

S 0094-9000(09)00763-7

Article electronically published on August 4, 2009

\title{
ON INVESTMENT AND MINIMIZATION OF SHORTFALL RISK FOR A DIFFUSION MODEL WITH JUMPS AND TWO INTEREST RATES VIA MARKET COMPLETION
}

UDC 519.21

\author{
SELLY KANE AND ALEXANDER MELNIKOV
}

\begin{abstract}
This paper deals with the problems of investment and shortfall risk minimization in the framework of a two-factor diffusion model with jumps and with different credit and deposit rates. The optimal strategies are derived by means of auxiliary completions of the initial market.
\end{abstract}

\section{INTRODUCTION}

In well-known financial market models one considers a unique interest rate for both deposit and credit (see Elliott and Kopp 10, Karatzas and Shreve 14, Föllmer and Leukert [12, Nakano [20]). In reality, the credit rate is always higher than the deposit rate. Such a market constraint brings new difficulties in the problems of hedging, investing and shortfall risk minimization (see Bergman 4, Kane and Melnikov [13, Korn [15, Bart [3], and also Cvitanić and Karatzas [8], Cvitanić [6, 7], Föllmer and Kramkov [11, Karatzas and Shreve [14, Cvitanić, Pham and Touzi [9], Soner and Touzi 21] regarding other market constraints).

As in Korn 15, Kane and Melnikov 13, we use a methodology of completions to solve the investment problem with logarithmic utility function and a shortfall risk minimization in a financial market consisting of a diffusion model with jumps and two interest rates.

\section{Description of the model AND Auxiliary RESUlts}

Let $\left\{\Omega, \mathcal{F}, F=\left(\mathcal{F}_{t}\right)_{t \geq 0}, \mathrm{P}\right\}$ be a standard stochastic basis. Assume there are two risky assets $S^{i}, i=1,2$, whose prices are described by the equations

$$
d S_{t}^{i}=S_{t^{-}}^{i}\left(\mu^{i} d t+\sigma^{i} d W_{t}-\nu^{i} d \Pi_{t}\right), \quad i=1,2 .
$$

Here $W$ is a standard Wiener process and $\Pi$ is a Poisson process with positive intensity $\lambda$. The filtration $F$ is generated by the independent processes $W$ and $\Pi, \mu^{i} \in \mathbf{R}, \sigma^{i}>0$, $\nu^{i}<1$.

We also assume that there are a deposit account $B^{1}$ and a credit account $B^{2}$ satisfying to

$$
d B_{t}^{i}=B_{t}^{i} r^{i} d t, \quad i=1,2 .
$$

2000 Mathematics Subject Classification. Primary 60H30, 62P05, 91B28; Secondary 60J75, 60G44, 91B30.

Key words and phrases. Constrained market, completion, hedging and pricing, diffusion with jumps, different interest rates.

The paper was supported by the discovery grant NSERC \#261855.

(C)2009 American Mathematical Society 
Denote by $\left(B^{1}, B^{2}, S^{1}, S^{2}\right)$ the market described by the above assets; any non-negative $\mathcal{F}_{T}$-measurable random variable $f_{T}$ is called a contingent claim with the maturity time $T$. In the $\left(B^{1}, B^{2}, S^{1}, S^{2}\right)$-market, a portfolio $\pi=\left(\beta^{1}, \beta^{2}, \gamma^{1}, \gamma^{2}\right)$ is an $\mathcal{F}_{t}$-predictable process, where we denote respectively by $\beta^{i}$ and $\gamma^{i}$ the number of units of the $i$ th bond and $i$ th stock in the wealth. The value of the portfolio $\pi$ is given by

$$
V_{t}=\beta_{t}^{1} B_{t}^{1}+\beta_{t}^{2} B_{t}^{2}+\gamma_{t}^{1} S_{t}^{1}+\gamma_{t}^{2} S_{t}^{2} \quad \text { a.s. }
$$

A portfolio $\pi$ is said to be self-financing if it satisfies the following property:

$$
d V_{t}=\beta_{t}^{1} d B_{t}^{1}+\beta_{t}^{2} d B_{t}^{2}+\gamma_{t}^{1} d S_{t}^{1}+\gamma_{t}^{2} d S_{t}^{2} \quad \text { a.s. }
$$

Such a portfolio will be said to be admissible if

$$
V_{t} \geq 0 \quad \text { a.s. for all } t \geq 0 \text {. }
$$

The set of admissible portfolios with initial capital $x$ is denoted by $\mathcal{A}(x)$.

The investor's position is identified with the wealth process $X_{t} \geq 0$. Here, the process $X$ is the capital of a self-financing and admissible portfolio. Under the above conditions and (2.1) $-(2.2)$, the wealth process $X_{t}$ has the form

$$
d X_{t}=X_{t^{-}}\left[\left(1-\alpha_{t}^{1}-\alpha_{t}^{2}\right)^{+} r^{1} d t-\left(1-\alpha_{t}^{1}-\alpha_{t}^{2}\right)^{-} r^{2} d t+\alpha_{t}^{1} \frac{d S_{t}^{1}}{S_{t^{-}}^{1}}+\alpha_{t}^{2} \frac{d S_{t}^{2}}{S_{t^{-}}^{2}}\right]
$$

Here $\alpha_{t}^{i}=\gamma_{t}^{i} S_{t^{-}}^{i} / X_{t^{-}}^{i}, i=1,2$, is the proportion of cash invested on the $i$ th stock in the wealth process, and $a^{+}=\max \{0, a\}, a^{-}=-\min \{0, a\}$.

Note that throughout the paper $\alpha$ will also be called a strategy. Further, the notation $X^{\pi}$ or $X^{\alpha}$ refers to the same wealth process.

Let us consider the special case where the financial market has the same deposit and credit rates: $r^{1}=r^{2}=r$, and hence, $B^{1}=B^{2}=B$. In the framework of such a $\left(B, S^{1}, S^{2}\right)$-market, the capital generated by an admissible portfolio process

$$
\pi:=\left(\beta, \gamma^{1}, \gamma^{2}\right)
$$

is described by

$$
\begin{gathered}
X_{t}=\beta_{t} B_{t}+\gamma_{t}^{1} S_{t}^{1}+\gamma_{t}^{2} S_{t}^{2} \quad \text { a.s. } \\
\frac{d X_{t}}{X_{t^{-}}}=\left[\left(1-\alpha_{t}^{1}-\alpha_{t}^{2}\right) r d t+\alpha_{t}^{1} \frac{d S_{t}^{1}}{S_{t^{-}}^{1}}+\alpha_{t}^{2} \frac{d S_{t}^{2}}{S_{t^{-}}^{2}}\right]
\end{gathered}
$$

If $\sigma^{1} \nu^{2} \neq \sigma^{2} \nu^{1}$, then the parameters

$$
\begin{gathered}
\phi=-\frac{\left(\mu^{1}-r\right) \nu^{2}-\left(\mu^{2}-r\right) \nu^{1}}{\sigma^{1} \nu^{2}-\sigma^{2} \nu^{1}} \\
\psi=\frac{\left(\mu^{1}-r\right) \sigma^{2}-\left(\mu^{2}-r\right) \sigma^{1}}{\sigma^{2} \nu^{1}-\sigma^{1} \nu^{2}} \lambda^{-1}-1
\end{gathered}
$$

define (see Melnikov et al. [17]) a density $Z$ of a unique martingale measure $P^{*}$ in the $\left(B, S^{1}, S^{2}\right)$-market as a stochastic exponent

$$
Z_{t}=\mathcal{E}_{t}(N)=\exp \left\{\phi W_{t}-\frac{\phi^{2}}{2} t+\left(\lambda-\lambda^{*}\right) t+\left(\ln \lambda^{*}-\ln \lambda\right) \Pi_{t}\right\},
$$

where $N_{t}=\phi W_{t}+\psi\left(\Pi_{t}-\lambda t\right)$. Under such a measure, the given Poisson process $\Pi$ has intensity $\lambda^{*}=\lambda(1+\psi)$ and $W_{t}^{*}=W_{t}-\phi t$ is a Wiener process.

We consider contingent claims of the form $f_{T}:=f\left(S_{T}^{1}\right)$.

Let us now turn to the $\left(B^{1}, B^{2}, S^{1}, S^{2}\right)$-market. 


\section{MAIN RESUlTS AND PRICING FORMUlAS}

To study the investment and shortfall risk minimization problems in the framework of a $\left(B^{1}, B^{2}, S^{1}, S^{2}\right)$-market we define a variety of $\left(B, S^{1}, S^{2}\right)\left(\right.$ or $\left.\left(B^{d}, S^{1}, S^{2}\right)\right)$-markets with the interest rates $r=r^{d}=r^{1}+d$, where $d=\left(d_{t}\right)$ is a predictable process such that $d_{t} \in\left[0, r^{2}-r^{1}\right]$.

Proposition 3.1. Let $d=\left(d_{t}\right)$ be a predictable process with values in the interval

$$
\left[0, r^{2}-r^{1}\right] \text {. }
$$

Assume that $\alpha_{d}:=\alpha=\left(\alpha^{1}, \alpha^{2}\right)$ generates the wealth processes $X^{\alpha, d}$ and $X^{\alpha}$ with initial capital $x$ in the $\left(B^{d}, S^{1}, S^{2}\right)$ and the $\left(B^{1}, B^{2}, S^{1}, S^{2}\right)$-markets respectively if further $\alpha_{d}$ satisfies

$$
\left(r^{2}-r^{1}-d_{t}\right)\left(1-\alpha_{t}^{1}-\alpha_{t}^{2}\right)^{-}+d_{t}\left(1-\alpha_{t}^{1}-\alpha_{t}^{2}\right)^{+}=0 .
$$

Then the above wealth processes coincide.

Proof of Proposition 3.1. Let $x$ be the initial capital associated to $\alpha$ in the $\left(B^{d}, S^{1}, S^{2}\right)$ market. If $\alpha$ satisfies (3.1), then we rewrite the latter equation as follows:

$$
\begin{aligned}
& \left(r^{2}-r^{1}-d_{t}\right)\left(1-\alpha_{t}^{1}-\alpha_{t}^{2}\right)^{-}+d_{t}\left(1-\alpha_{t}^{1}-\alpha_{t}^{2}\right)^{+} \\
& \quad=r^{2}\left(1-\alpha_{t}^{1}-\alpha_{t}^{2}\right)^{-}-\left(r^{1}+d_{t}\right)\left(1-\alpha_{t}^{1}-\alpha_{t}^{2}\right)^{-}+d_{t}\left(1-\alpha_{t}^{1}-\alpha_{t}^{2}\right)^{+} \\
& \quad=r^{2}\left(1-\alpha_{t}^{1}-\alpha_{t}^{2}\right)^{-}-r^{d}\left(1-\alpha_{t}^{1}-\alpha_{t}^{2}\right)-r^{1}\left(1-\alpha_{t}^{1}-\alpha_{t}^{2}\right)^{+}=0 .
\end{aligned}
$$

Hence,

$$
r^{d}\left(1-\alpha_{t}^{1}-\alpha_{t}^{2}\right)=r^{1}\left(1-\alpha_{t}^{1}-\alpha_{t}^{2}\right)^{+}-r^{2}\left(1-\alpha_{t}^{1}-\alpha_{t}^{2}\right)^{-}
$$

and

$$
\begin{aligned}
\frac{d X_{t}^{\alpha, d}}{X_{t^{-}}^{\alpha, d}} & =r^{d}\left(1-\alpha_{t}^{1}-\alpha_{t}^{2}\right) d t+\alpha_{t}^{1} \frac{d S_{t}^{1}}{S_{t^{-}}^{1}}+\alpha^{2} \frac{d S_{t}^{2}}{S_{t^{-}}^{2}} \\
& =\left(r^{1}\left(1-\alpha_{t}^{1}-\alpha_{t}^{2}\right)^{+}-r^{2}\left(1-\alpha_{t}^{1}-\alpha_{t}^{2}\right)^{-}\right) d t+\alpha^{1} \frac{d S_{t}^{1}}{S_{t^{-}}^{1}}+\alpha^{2} \frac{d S_{t}^{2}}{S_{t^{-}}^{2}} \\
& =\frac{d X_{t}^{\alpha}}{X_{t^{-}}^{\alpha}}
\end{aligned}
$$

Therefore, the wealth processes $X_{t}^{\alpha, d}(x)$ and $X_{t}^{\alpha}(x)$ on respectively $\left(B^{d}, S^{1}, S^{2}\right)$ and $\left(B^{1}, B^{2}, S^{1}, S^{2}\right)$-markets coincide.

3.1. Investment problem. In parallel to the hedging agent who wants to find the optimal strategy to hedge his claim $f_{T}$ (see Kane and Melnikov [13]), an investing agent has to find the optimal strategy that allows him to maximize the expected utility of his terminal wealth. Assume a given utility function $U: \mathbf{R}_{+} \rightarrow \mathbf{R}$ is concave, non-decreasing, continuously differentiable, and such that

$$
\begin{aligned}
& \lim _{x \rightarrow \infty} U^{\prime}(x)=0, \\
& \lim _{x \rightarrow 0} U^{\prime}(x)=\infty .
\end{aligned}
$$

Let $u(x)$ be the cost function in the $\left(B^{d}, S^{1}, S^{2}\right)$-market. The investment problem (see, for instance, Karatzas and Shreve [14], Melnikov et al. [17]) consists in finding

$$
\begin{aligned}
u(x) & =\sup _{\pi \in S F} \mathrm{E}\left(U\left(X_{T}^{\pi, d}(x)\right)\right)=\mathrm{E}\left(U\left(X_{T}^{\pi^{*}, d}(x)\right)\right) \\
& =\sup _{Y \in \chi} \mathrm{E}\left(U\left(Y_{T}(x)\right)\right)=\mathrm{E}\left(U\left(Y_{T}^{*}(x)\right)\right),
\end{aligned}
$$


where $\chi=\left\{Y\right.$ positive: $\left.Y_{t}(x)=x+\int_{0}^{t} \gamma_{u} d \tilde{X}_{s}\right\}, \gamma$ is a predictable process and $\tilde{X}$ is a $P^{*}$ local martingale. To solve the problem, we introduce $V(y)$, the conjugate function of $U(x)$, whose relations to the latter are given by

$$
\begin{array}{ll}
V(y)=\sup _{x>0} U(x)-x y, & y>0, \\
U(x)=\inf _{y>0} V(y)+x y, & x>0 .
\end{array}
$$

We denote $I(x)=\left(\left(U^{\prime}\right)^{-1}(x)\right)=-V^{\prime}(x), y_{0}=\inf \{y: v(y)<\infty\}$, and

$$
x_{0}=\lim _{y \rightarrow y_{0}}\left(-v^{\prime}(y)\right) .
$$

The functions $v(y)$ and $u(x)$ are such that:

1) the function $u(x)<\infty$ is continuously differentiable for all $x>0$, strictly concave on $\left(0, x_{0}\right)$, and the function $v(x)<\infty$ is continuously differentiable for all $y>0$, strictly convex on $\left(y_{0}, \infty\right)$ with

$$
\begin{array}{ll}
v(y)=\sup _{x>0} u(x)-x y, & y>0, \\
u(x)=\inf _{y>0} v(y)+x y, & x>0 .
\end{array}
$$

2) If $y=u^{\prime}(x)$, where $x<x_{0}$ and $y<y_{0}$, then the optimal solution of (3.2) is

$$
Y_{T}^{*}(x)=I\left(y Z_{T}\right) .
$$

We consider the case $U(x)=\ln (x)$ in the $\left(B^{d}, S^{1}, S^{2}\right)$ financial market. Substituting $U(x)$ in (3.3), we derive $V(y)=-\ln (y)-1$ and

$$
\begin{aligned}
v(y) & =\mathrm{E}\left[V\left(y Z_{T}\right)\right]=-\mathrm{E}\left[\ln \left(y Z_{T}\right)\right]-1=-\ln (y)-1-\mathrm{E}\left[\ln \left(Z_{T}\right)\right] \\
& =-\ln (y)-1+\frac{\phi^{2}}{2} T-\left(\ln \left(\lambda^{*}\right)-\ln (\lambda)\right) \lambda T+\left(\lambda^{*}-\lambda\right) T .
\end{aligned}
$$

We substitute the above expression of $v(y)$ into equality (3.6) and find the price function

$$
u(x)=\ln (x)+\frac{\phi^{2}}{2} T-\left(\ln \left(\lambda^{*}\right)-\ln (\lambda)\right) \lambda T+\left(\lambda^{*}-\lambda\right) T .
$$

Next, we derive the optimal proportions invested in the different assets involved. From equation (3.7), we know that

$$
\begin{aligned}
Y_{T}^{*}(x) & =\frac{X_{T}^{\pi^{*}, d}}{B_{T}}=I\left(y Z_{T}\right)=\frac{1}{y Z_{T}}=\frac{x}{Z_{T}} \\
& =x \exp \left\{-\phi W_{T}+\frac{\phi^{2}}{2} T-\Pi_{T} \ln \left(\frac{\lambda^{*}}{\lambda}\right)+\left(\lambda^{*}-\lambda\right) T\right\}
\end{aligned}
$$

and solving (2.7) for $\alpha_{t}=\alpha$ yields

$$
\begin{aligned}
\frac{X_{T}^{\pi, d}}{B_{T}^{d}}=x \exp \left\{\left(\alpha^{1}\left(\mu^{1}-r_{d}\right)+\alpha^{2}\left(\mu^{2}-r_{d}\right)-\frac{\left(\alpha^{1} \sigma^{1}+\alpha^{2} \sigma^{2}\right)^{2}}{2}\right) T\right. \\
\left.+\left(\alpha^{1} \sigma^{1}+\alpha^{2} \sigma^{2}\right) W_{T}+\ln \left(1-\alpha^{1} \nu^{1}-\alpha^{2} \nu^{2}\right) \Pi_{T}\right\} .
\end{aligned}
$$

We identify expressions (3.9) and (3.10) and obtain the values for $\alpha^{1}$ and $\alpha^{2}$ :

$$
\alpha^{1}=\frac{\phi \nu^{2}-\sigma^{2}\left(\lambda / \lambda^{*}-1\right)}{\nu^{1} \sigma^{2}-\nu^{2} \sigma^{1}}, \quad \alpha^{2}=\frac{\phi \nu^{1}-\sigma^{1}\left(\lambda / \lambda^{*}-1\right)}{\nu^{2} \sigma^{1}-\nu^{1} \sigma^{2}} .
$$

In the $\left(B^{d}, S^{1}, S^{2}\right)$-market, the optimal proportions invested are $\alpha^{1}$ on the first stock, $\alpha^{2}$ on the second stock and the rest $\left(1-\alpha^{1}-\alpha^{2}\right)$ on the bank account. 
Let us turn to the two-interest-rates financial market $\left(B^{1}, B^{2}, S^{1}, S^{2}\right)$. The previous results lead to the following theorem.

Theorem 3.1. Let the wealth processes $X_{t}^{\pi, d}(x)$ and $X_{t}^{\pi}(x)$ in the $\left(B^{d}, S^{1}, S^{2}\right)$ and $\left(B^{1}, B^{2}, S^{1}, S^{2}\right)$ financial markets satisfy (2.5) and (2.7), respectively, and let relation (3.1) hold for the optimal proportions $\alpha_{t}$ of problem (3.2) in the $\left(B^{d}, S^{1}, S^{2}\right)$-market. Then considering a logarithmic utility function, we obtain in the $\left(B^{1}, B^{2}, S^{1}, S^{2}\right)$-market that

1) the price function $u(x)$ is given by (3.8), and

2) the optimal proportions invested in the different assets are $\alpha^{1}$ on $S^{1}, \alpha^{2}$ on $S^{2}$, $\left(1-\alpha^{1}-\alpha^{2}\right)^{+}$on the deposit account (if $\left.\left(1-\alpha^{1}-\alpha^{2}\right)>0\right)$ and $\left(1-\alpha^{1}-\alpha^{2}\right)^{-}$ on the credit account (if $\left(1-\alpha^{1}-\alpha^{2}\right)<0$ ).

Proof. Let $\alpha^{*}$, the optimal proportions in the $\left(B^{d}, S^{1}, S^{2}\right)$-market, satisfy (3.1); then $\alpha^{*}$ is optimal for the $\left(B^{1}, B^{2}, S^{1}, S^{2}\right)$-market.

For any strategy $\pi$, we have $X_{t}^{\pi}(x) \leq X_{t}^{\pi, d}(x)$ and

$$
\sup _{\pi \in S F} \mathrm{E}\left[U\left(X_{T}^{\pi}(x)\right)\right] \leq \sup _{\pi \in S F} \mathrm{E}\left[U\left(X_{T}^{\pi, d}(x)\right)\right]=\mathrm{E}\left[U\left(X_{T}^{\pi^{*}, d}(x)\right)\right] \stackrel{\sqrt[3.1 x]{=}}{\mathrm{E}}\left[U\left(X_{T}^{\pi^{*}}(x)\right)\right] .
$$

Hence

$$
\sup _{\pi \in S F} \mathrm{E}\left[U\left(X_{T}^{\pi}(x)\right)\right]=\mathrm{E}\left[U\left(X_{T}^{\pi^{*}}(x)\right)\right]=\mathrm{E}\left[U\left(X_{T}^{\pi^{*}, d}(x)\right)\right]=u(x) .
$$

Regarding the optimal proportions in the $\left(B^{1}, B^{2}, S^{1}, S^{2}\right)$-market, we derive from (3.1) and (3.12) that

$$
X_{T}^{\pi^{*}}(x)=X_{T}^{\pi^{*}, d}(x)=Y_{T}^{*, d}(x) e^{r^{d} T} .
$$

Solving (2.5) for $X_{T}^{\pi^{*}}(x)$ and identifying the latter with $Y_{T}^{*, d} e^{r^{d} T}$, the optimal proportions invested in the $\left(B^{1}, B^{2}, S^{1}, S^{2}\right)$-market are $\alpha^{1}$ on $S^{1}$ and $\alpha^{2}$ on $S^{2}$. Since $\alpha^{1}$ and $\alpha^{2}$ are constants, we invest $\left(1-\alpha^{1}-\alpha^{2}\right)^{+}$on $B^{1}$ if $\left(1-\alpha^{1}-\alpha^{2}\right)>0$, and we invest $-\left(1-\alpha^{1}-\alpha^{2}\right)^{-}$ on $B^{2}$ if $\left(1-\alpha^{1}-\alpha^{2}\right)<0$.

Consider a particular case $\sigma^{1}=0, \nu^{2}=0$ and assume $\left(1-\alpha^{1}-\alpha^{2}\right) \geq 0$ (only the lending rate $r^{1}$ is applicable). Then, the market $\left(B^{d}, S^{1}, S^{2}\right)$ is defined by

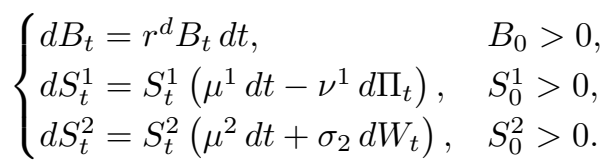

From relation (2.8) we obtain that

$$
\phi=-\frac{\mu^{2}-r^{1}}{\sigma_{2}}, \quad \lambda^{*}=\frac{\mu^{1}-r^{1}}{\nu^{1}} .
$$

Exploiting (3.14) and (3.11), we derive

$$
\alpha^{1}=\frac{\mu^{1}-r^{1}-\lambda \nu^{1}}{\nu^{1}\left(\mu^{1}-r\right)}, \quad \alpha^{2}=\frac{\mu^{2}-r^{1}}{\left(\sigma_{2}\right)^{2}} .
$$

Note that $\alpha_{1}$ and $\alpha_{2}$ are the Merton points in a pure jump and pure diffusion model, respectively. In the setting of a two-interest-rates financial market with the above assumptions, the optimal proportions invested are defined by (3.15) for $S^{1}$ and $S^{2}$ correspondingly, and $\left(1-\alpha^{1}-\alpha^{2}\right)$ in $B^{1}$ and 0 in $B^{2}$. 
3.2. Minimization of a shortfall risk. In the $\left(B^{d}, S^{1}, S^{2}\right)$-market, we found in Kane and Melnikov [13. the optimal strategy and initial capital required to hedge perfectly a claim $f\left(S_{T}^{1}\right)$. We also derived in the previous section the optimal investment strategy and terminal wealth of an expected utility maximization problem.

Now we consider an investor whose initial capital $x$ is less than the required

$$
E^{d, *}\left[e^{-r^{d} T} f_{T}\right] .
$$

In such a case, a perfect hedge is no longer possible, but one can minimize the risk of shortfall given the initial constraint on the cost:

$$
u(x)=\inf _{\substack{\pi \in \mathcal{A}, x<E^{d, *}\left[f\left(S_{T}^{1}\right) e^{-r^{d} T}\right]}} \mathrm{E}\left[l_{p}\left(\left(f_{T}-X_{T}^{\pi, d}(x)\right)^{+}\right)\right] .
$$

Note that $u(x)$ is different from that in Section 3.1. The loss function is $l_{p}(x)=x^{p} / p$ with $p>1, \mathcal{A}=\left\{\pi: \mathrm{E}\left[\sup _{0 \leq t \leq T}\left|X_{t}^{\pi}(0)\right|\right]<\infty\right\}$ and $f_{T} \in L^{p+\varepsilon}\left(\Omega, \mathcal{F}_{T}, \mathrm{P}\right)$ for some $\varepsilon>0$. From Nakano [20, the solution of the problem (3.16) in the setting of a $\left(B^{d}, S^{1}, S^{2}\right)$ market consists in finding the perfect hedge of the claim $f_{T}$ and the optimal strategy for an expected utility maximization problem (Section 3.2 helps in solving this part; see also Karatzas-Shreve [14, Nakano [20]). Such a solution is characterized by what follows.

1) The optimal solution for (3.16) is $\pi^{*}=\pi_{f_{T}}-\pi_{0}$, where $\pi_{f_{T}}$ is the perfect hedge for $f_{T}$ and $\pi_{0}$ is the optimal strategy for

$$
J(z):=\inf _{\pi \in \mathcal{A}_{0}(z)} \mathrm{E}\left[l_{p}\left(X_{T}^{\pi, d}(z)\right)\right]
$$

with $z=x_{f_{T}}-x$. We denote $\mathcal{A}_{0}(z)=\left\{\pi \in \mathcal{A}\right.$ and $X_{t}^{\pi} \geq 0, t \in[0, T]$ a.s. $\}$.

2) Let $\alpha_{0}(t)$ be the optimal portfolio proportions associated to the solution $\pi_{0}$ of (3.17). Then $\alpha_{0}(t):=\alpha_{0}=\left(\alpha_{0}^{1}, \alpha_{0}^{2}\right)$ of $J(z)$ is given by

$$
\alpha_{0}^{1}=\frac{\frac{\phi \nu^{2}}{p-1}+\sigma^{2}\left(\frac{\lambda^{*}}{\lambda}\right)^{q-1}}{\nu^{2} \sigma^{1}-\nu^{1} \sigma^{2}}, \quad \alpha_{0}^{2}=\frac{\frac{\phi \nu^{1}}{p-1}+\sigma^{1}\left(\frac{\lambda^{*}}{\lambda}\right)^{q-1}}{\sigma^{2} \nu^{1}-\sigma^{1} \nu^{2}},
$$

where $q$ is such that $1 / p+1 / q=1$.

3) The price function is

$$
u(x)=l_{p}\left(x_{f_{T}}-x\right) e^{(-(p-1) a T)},
$$

with

$$
a=-q r^{d}+\frac{1}{2} q(q-1) \phi^{2}-\lambda\left((q-1)-q\left(\frac{\lambda^{*}}{\lambda}\right)+\left(\frac{\lambda^{*}}{\lambda}\right)^{q}\right) .
$$

4) The optimal terminal wealth is given by

$$
X_{T}^{\pi_{f_{T}}-\pi_{0}, d}(x)=f_{T}-\left(x_{f_{T}}-x\right)\left(Z_{T}\right)^{q-1} \exp \left\{\left(-\left(a+\frac{r^{d}}{p-1}\right) T\right)\right\} .
$$

Consider now the identical problem in a two-interest-rates financial market:

$$
u(x)=\inf _{\substack{\pi \in \mathcal{A} \\ x<C_{-}}} \mathrm{E}\left[l_{p}\left(\left(f_{T}-X_{T}^{\pi}(x)\right)^{+}\right)\right] .
$$

We give the solution of the problem (3.20).

Theorem 3.2. Let $X_{t}^{\pi, d}(x)$ and $X_{t}^{\pi}(x)$ be the wealth processes in the $\left(B^{d}, S^{1}, S^{2}\right)$ and $\left(B^{1}, B^{2}, S^{1}, S^{2}\right)$-markets, and let the initial capital $x$ satisfy respectively relations (2.5) and (2.7). Further, assume that the optimal proportion $\alpha_{t}$ in the $\left(B^{d}, S^{1}, S^{2}\right)$-market 
satisfies (3.1). Assume also that the optimal strategy $\alpha_{f_{T}}$ hedging $f_{T}$ in the $\left(B^{d}, S^{1}, S^{2}\right)$ market satisfies the conditions provided in Proposition 3.1.

Then, in the $\left(B^{1}, B^{2}, S^{1}, S^{2}\right)$-market,

1) the price function (3.20) is given by equation (3.19),

2) the optimal proportions invested are

$$
\begin{array}{ll}
\alpha_{t}^{1}=\frac{\alpha_{f}^{1} X_{t^{-}}^{\pi_{f_{T}}}\left(x_{f_{T}}\right)-\alpha_{0}^{1} X_{t^{-}}^{\pi_{0}}\left(x_{f_{T}}-x\right)}{X_{t^{-}}^{\pi_{f_{T}}-\pi_{0}}(x)} & \text { on } S^{1}, \\
\alpha_{t}^{2}=\frac{\alpha_{f}^{2} X_{t^{-}}^{\pi_{f_{T}}}\left(x_{f_{T}}\right)-\alpha_{0}^{2} X_{t^{-}}^{\pi_{0}}\left(x_{f_{T}}-x\right)}{X_{t^{-}}^{\pi_{f_{T}}-\pi_{0}}(x)} & \text { on } S^{2},
\end{array}
$$

and $\left(1-\alpha_{t}^{1}-\alpha_{t}^{2}\right)^{+}$on the deposit account and $\left(1-\alpha_{t}^{1}-\alpha_{t}^{2}\right)^{-}$on the credit account.

Proof. Let the optimal proportions $\alpha_{t}^{*}$ invested for the problem (3.16) in the $\left(B^{d}, S^{1}, S^{2}\right)$ market satisfy (3.1). Then,

$$
X_{t}^{\pi^{*}, d}(x)=X_{t}^{\pi^{*}}(x) \text { for all } 0 \leq t \leq T
$$

and $\alpha^{*}$ is optimal for the same problem in the $\left(B^{1}, B^{2}, S^{1}, S^{2}\right)$ financial market.

The $\left(B^{1}, B^{2}, S^{1}, S^{2}\right)$-market admits a higher deposit rate and a lower lending rate than the $\left(B^{d}, S^{1}, S^{2}\right)$-market. Therefore, for any strategy $\pi, X_{t}^{\pi}(x) \leq X(x)_{t}^{\pi, d}$ and from the monotonicity of $l_{p}$ it follows that

$$
\begin{aligned}
\inf _{\pi \in \mathcal{A},\left\{x<E^{d, *}\left[e^{-r^{d} T} f_{T}\right]\right\}} \mathrm{E}\left[l_{p}\left(\left(f_{T}-X_{T}^{\pi, d}(x)\right)^{+}\right)\right] \\
\quad \leq \inf _{\pi \in \mathcal{A},\left\{x<E^{d, *}\left[e^{-r^{d} T} f_{T}\right]\right\}} \mathrm{E}\left[l_{p}\left(\left(f_{T}-X_{T}^{\pi}(x)\right)^{+}\right)\right] .
\end{aligned}
$$

Now if $\alpha^{*}$ is optimal for the left hand side of inequality (3.21) and satisfies equality (3.1), then it is also optimal for the right hand side.

The price function $u(x)$ is given by

$$
\begin{aligned}
u(x) & =\inf _{\pi \in \mathcal{A},\left\{x<E^{d, *}\left[e^{-r^{d} T} f_{T}\right]\right\}} \mathrm{E}\left[l_{p}\left(\left(f_{T}-X_{T}^{\pi, d}(x)\right)^{+}\right)\right] \\
& =\mathrm{E}\left[l_{p}\left(\left(f_{T}-X_{T}^{\pi^{*}, d}(x)\right)^{+}\right)\right] \\
& =\mathrm{E}\left[l_{p}\left(\left(f_{T}-X_{T}^{\pi^{*}}(x)\right)^{+}\right)\right] \\
& =\inf _{\pi \in \mathcal{A},\left\{x<E^{d, *}\left[e^{-r^{d} T} f_{T}\right]\right\}} \mathrm{E}\left[l_{p}\left(\left(f_{T}-X_{T}^{\pi}(x)\right)^{+}\right)\right] .
\end{aligned}
$$

Since the optimal proportion $\alpha_{f_{T}}$ hedging $f_{T}$ satisfies the conditions of Proposition 3.1 (i.e. $C_{-}=C_{r^{d}}$ ), we obtain

$$
u(x)=\inf _{\left\{\pi \in \mathcal{A},\left\{x<C_{-}\right\}\right\}} \mathrm{E}\left[l_{p}\left(\left(f_{T}-X_{T}^{\pi}(x)\right)^{+}\right)\right] .
$$

The optimal terminal wealth is $X_{T}^{\pi}(x)=X_{T}^{\pi, d}(x)=X_{T}^{\pi_{f}}\left(x_{f}\right)-X_{T}^{\pi_{0}}\left(x-x_{f}\right)$.

The optimal proportions invested are given by

$$
\begin{gathered}
\pi=\pi_{f}-\pi_{0}, \\
\gamma_{t} S=\gamma_{t, f} S-\gamma_{t, 0} S, \\
\alpha_{t} X_{t^{-}}^{\pi}=\alpha_{t, f} X_{t^{-}}^{\pi_{f}}-\alpha_{t, 0} X_{t^{-}}^{\pi_{0}}
\end{gathered}
$$




\section{BIBLIOGRAPHY}

1. K. K. Aase, Contingent claim valuation when the security price is a combination of an Ito process and a random point process, Stochastic Process. Appl. 28 (1988), 185-220. MR952829 (89k:90015)

2. J. Bardhan and X. Chao, Pricing options on securities with discontinuous returns, Stochastic Process. Appl. 48 (1993), 123-137. MR1237171 (94g:90011)

3. Y. Bart, Option hedging in the binomial model with differing interest rates, Uspekhi Math. Nauk 53 (1998), no. 5, 227-228; English transl. in Russian Math. Surveys 53 (1998), 10841085. MR 1691190

4. Y. Bergman, Option pricing with different interest rates for borrowing and for lending, Working Paper, University of California, Berkeley 109 (1981).

5. D. Colwell and R. Elliott, Discontinuous asset prices and non-attainable contingent claims and corporate policy, Math. Finance 3 (1993), 295-318.

6. J. Cvitanić, Optimal trading under constraints, Lectures Notes in Mathematics, vol. 1656, Springer-Verlag, Berlin, 1997, pp. 123-190. MR1478201

7. J. Cvitanić, Theory of Portfolio Optimization in Markets with Frictions, Handbooks in Math. Finance: Option Pricing, Interest Rates and Risk Management (E. Jouini and M. Musiela, eds.), Cambridge University Press, 2001. MR1848563

8. J. Cvitanić and I. Karatzas, Hedging contingent claims with constrained portfolio, Annals Appl. Probab. 3(3) (1993), 652-681. MR1233619 (95c:90022)

9. J. Cvitanić, H. Pham, and N. Touzi, Super-replication in stochastic volatility models under portfolio constraints, J. Appl. Probab. 36 (1999), 523-545. MR.1724796 (2001a:91048)

10. R. Elliott and P. E. Kopp, Mathematics of Financial Markets, Springer-Verlag, Berlin, 1998. MR 2098795 (2005g:91001)

11. H. Föllmer and D. O. Kramkov, Optional decompositions under constraints, Probab. Theory Related Fields 109 (1997), 1-25. MR:1469917(98j:60065)

12. H. Föllmer and P. Leukert, Efficient hedging: Cost versus shortfall risk, Finance Stoch. 4 (2000), 117-146. MR1780323 (2001f:91054)

13. S. Kane and A. Melnikov, On pricing contingent claims in a two interest rates jump diffusion model via market completions, Theory Probab. Math. Statist. 77 (2007), 57-69. MR2432772 (2009f:91062)

14. I. Karatzas and S. Shreve, Methods of Mathematical Finance, Springer-Verlag, New York, 1998. MR.1640352 (2000e:91076)

15. R. Korn, Contingent claim valuation in a market with different interest rates, Math. Methods Oper. Res. 42 (1995), 255-274. MR 1358829

16. R. Krutchenko and A. V. Melnikov, Quantile hedging for a jump-diffusion financial market, Trends in Mathematics (M. Kohlmann, ed.), Birkhäuser Verlag, Basel/Switzerland, 2001, pp. 215-229. MR 1882833

17. A. V. Melnikov, M. Nechaev, and S. Volkov, Mathematics of Financial Obligations, American Mathematical Society, Providence, R.I., 2002. MR1918716 (2003f:91055)

18. F. Mercurio and W. Runggaldier, Option pricing for jump-diffusions: Approximations and their interpretation, Math. Finance 3 (1993), 191-200.

19. R. C. Merton, Continuous-time finance, Basil-Blackwell, Oxford, 1990.

20. Y. Nakano, Minimization of shortfall risk in a jump-diffusion model, Statist. Probab. Lett. 67 (2004), 87-95. MR2039936 (2005b:62137)

21. H. Soner and N. Touzi, Superreplication under gamma constraints, J. Control Optim. 39 (2000), 73-96. MR1780909 (2002h:91068)

Department of Mathematical and Statistical Sciences, University of Alberta, Edmonton, T6G2G1 CANADA

E-mail address: skane@ualberta.ca

Department of Mathematical and Statistical Sciences, University of Alberta, Edmonton, T6G2G1 CANADA

E-mail address: melnikov@ualberta.ca

Received 9/JAN/2007

Translated by THE AUTHORS 